\title{
Geometrical checking by virtual gauge, including measurement uncertainties
}

\author{
J. Mailhe ${ }^{\mathrm{a}}$, J.M. Linares ${ }^{\mathrm{a}}$, J.M. Sprauel ${ }^{\mathrm{a}}$, P. Bourdet (1) ${ }^{\mathrm{b}, *}$ \\ ${ }^{a}$ EA(MS) ${ }^{2}$, Mediterranean University-I.U.T., Avenue Gaston Berger, 13625 Aix en Provence Cedex 1, France \\ ${ }^{\mathrm{b}}$ E.N.S. Cachan, 61 Avenue Président Wilson, 94230 Cachan, France
}

For the most part, metrology software is currently based on the calculation of distances or angles between geometrical elements. This kind of method implies series of geometrical constructions which are amplifying measurement uncertainties. Usually, the estimation of error bars is then done after the geometrical verification. Hence, uncertainties are not directly taken into account during the checking process. To avoid these impediments, a new checking method, based on virtual gauges, is proposed in this paper. Based on Gram matrix, virtual gauges permit to perform checking without any intermediate geometrical construction. Moreover, thanks to a statistical description of the specified surface, the measurement uncertainties are thus directly taken into account during the conformance test process. An example of application is presented to demonstrate the relevance of this approach.

\section{Introduction}

Since the last 10 years, progress has been completed in specification and verification of geometrical products. In metrology, the control of measurement uncertainties has thus been an active field [1-4]. Simultaneously, ISO standards proposed a new specification language called GPS. This language, based on the notions of tolerance zone and modifiers (maximum material condition (MMC), least material condition (LMC), envelop requirement (E), etc.), permits defining a set of acceptable part geometries [5]. The verification process shall include these two aspects. Currently, the tests implemented in major part of metrology software are based on the calculation of angles and distances. No uncertainty is however taken into account, but only mean values are usually evaluated for checking. Moreover, this kind of method uses series of geometrical constructions which amplify the initial measurement uncertainties [6]. When error bars are provided, it is done at the end of the verification process. The feature tolerance zone permits using a new kind of test based on a virtual gauge $[7,8]$. However, current works performed on virtual gauge do not integrate measurement uncertainties. The aim of this paper is therefore to present a new verification method based on a virtual gauge and a statistical representation of real surfaces including measurement uncertainties.

The principle of this method is presented in Fig. 1. As shown on the top left corner of the figure, a set of measured points is first acquired by a Coordinate Measuring Machine. This permits expressing a field of probability of presence of the matter (FPPM) which defines the probability of any point of the three-dimensional space of being located inside the matter. On the right side of the figure, a virtual gauge is then generated with respect to the

* Corresponding author. geometrical specifications. In the bottom of the figure the conformance test is finally performed by comparing the virtual gauge to the FPPM. This comparison is carried out through the construction of an interference probability map (IPM), which provides the probability of any point of the real surfaces to fit the required gauge boundaries. To explain this global procedure, our paper will be split in the three sections presented in Fig. 2.

First, the principle of construction of virtual gauges will be presented. Second, the FPPM model will be explained. Finally, the calculation of IPMs will be clarified, and the problem of optimization of the virtual gauge position will be detailed.

\section{Virtual gauge construction}

As written before, GPS language uses tolerance zones and virtual states to express geometrical conditions. The boundaries of any specified three-dimensional space domain can thus be represented by elementary virtual gauges. Virtual gauges are the numerical and extended version of physical ones. Each elementary gauge, of index $i$, can be represented by a family of seven vectors, named $\mathrm{E}_{\mathrm{i}}$, see the following equation.

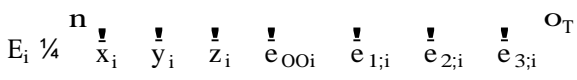

The first three vectors form the construction basis which will permit expressing the link between all elementary gauges. Fourth vector defines the translation direction of the gauge. The last three vectors form the local basis which characterizes the orientation of the gauge. Fig. 3 represents a virtual gauge system connected by the construction basis.

The vector family of an elementary gauge can be efficiently modeled by a Gram matrix $\mathrm{G}_{\mathrm{i}}$ [9] defined by the following tensorial 


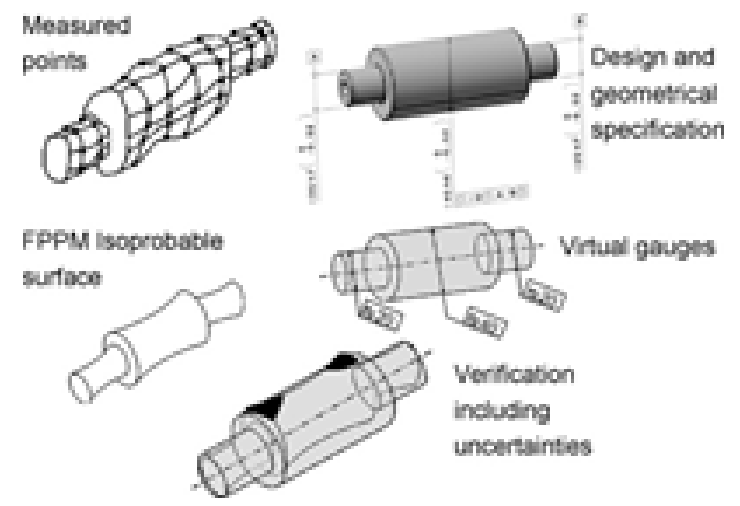

Fig. 1. Geometrical checking by virtual gauge.

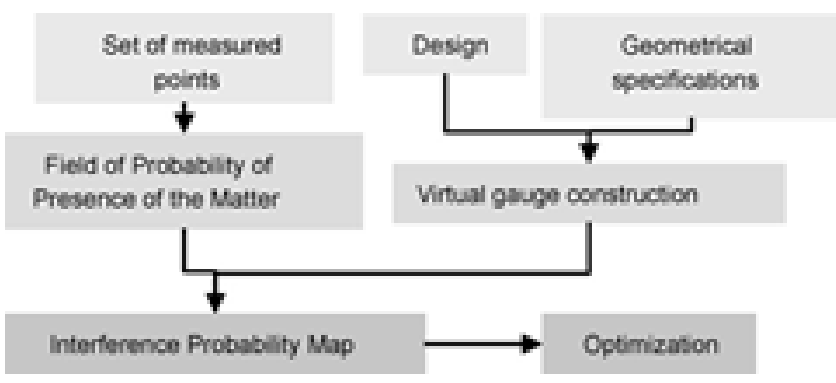

Fig. 2. Checking procedure using a virtual gauge.

product (2):

$\mathrm{G}_{\mathrm{i}} 1 / 4 \mathrm{E}_{\mathrm{i}} \quad \mathrm{i}$

$\mathrm{E}^{\mathrm{T}}$

Gram matrix $G_{i}$ is a second-order tensor of rank 3 (threedimensional space) and size 717 . The construction of the global virtual gauge requires a connection between the different elementary entities (Fig. 3). This condition is realized by jimposing the equality of the entire construction basis $\bar{x}_{i} ; \bar{y}_{i} ; \bar{z}_{i}$. It is performed by a connection operator $\mathrm{C}$ which is representing all connection specifications (3).

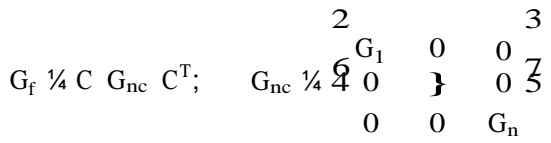

Tensor $G_{n c}$ represents the disconnected global virtual gauge constructed with the whole (number $n$ ) elementary entities. The linear operator $C$ is a matrix of size $71 \mathrm{n}$. The final Gram matrix $G_{1}$ represents the global virtual gauge. It is a second-order tensor, still of rank 3 , but of size $(71 \mathrm{n}) 1(71 \mathrm{n})$. The result of the connection

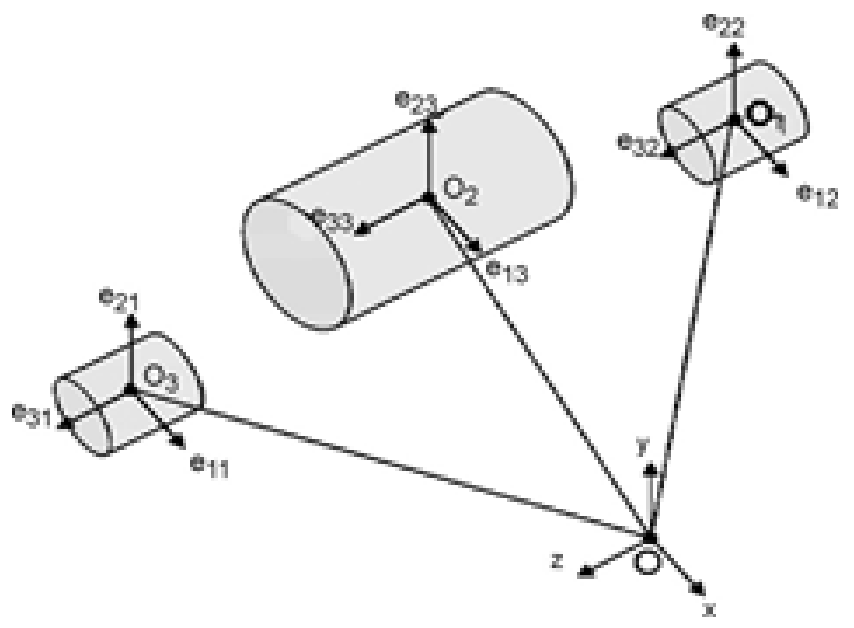

Fig. 3. Virtual gauge assembly.

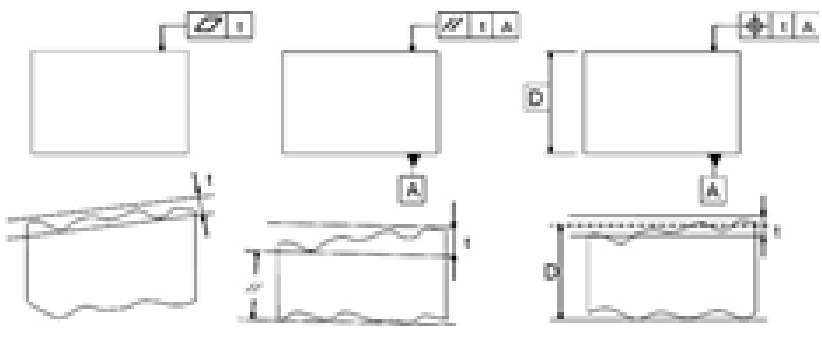

Fig. 4. DOF of tolerance zones or gauges.

is a global virtual gauge given in a specific position. However tolerance zones can have their own degrees of freedom (DOF) (Fig. 4).

To model these DOFs, a perturbation operator is introduced [10]. The principle of the perturbation is to express a variation of the vector family and there after to derive the resulting Gram matrix (4).

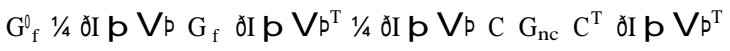

Matrix $V$ represents the variations $D_{i}$ applied to the different vector families $E_{i}$. Hence the following relation is obtained (5):

$$
\begin{aligned}
& { }^{2} \mathrm{DE}_{1}{ }^{3} \quad 2 \mathrm{E}_{1}{ }^{3}
\end{aligned}
$$

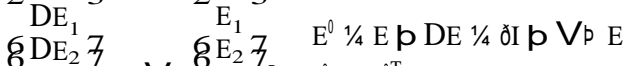

$$
\begin{aligned}
& \left.4: 5^{1 / 4} \mathrm{~V} 66^{2} \cdot 7_{5}\right) \mathrm{G}^{0} 1 / 4 \mathrm{E}^{0 \mathrm{~T}} \\
& \mathrm{E}^{0} \mathrm{DE} \\
& \text { E } \\
& G^{0} 1 / 4 \text { I p Vp G }
\end{aligned}
$$

where $E$ represents the global family of vectors and $E^{0}$ the perturbed result.

The last step of the virtual gauge construction is the expression of its surface boundaries. Such surface will be characterized by a parametric equation Sou; vp based on the local basis of each elementary virtual gauge and curvilinear coordinates, u and v. A formulation of this parametric equation, based on the Gram matrix, can be obtained by introducing a vector $\mathrm{L}$ of geometrical parameters related to the curvilinear coordinates (6).

Sðu;vp $1 / 4 \mathrm{G}_{\mathrm{f}}$ Lðu; vp

\section{Field of probability of the presence of matter}

Any measurement is tainted by uncertainties related to different sources: geometry and thermal expansion compensation errors, form defects not described in the surface model choice sampling strategy, etc. These uncertainties will be propagated when estimating the best fitted surface. As a first approach, the instrument errors (geometry, thermal expansion deviations, etc.) are however assumed completely corrected and their uncertainties neglected. Nevertheless, in our verification method, a real surface is not just represented by a mean feature, but is characterized by a full random vector. This last consists of centre point coordinates and vector components representing the derived element and intrinsic parameters such as radii and apgles. A best fitting of the acquired points provides a mean vector $\mathrm{P}$ and a covariance matrix $\operatorname{Cov} \partial \mathrm{P} P[11]$. At this time, an iterative non-linear least squares

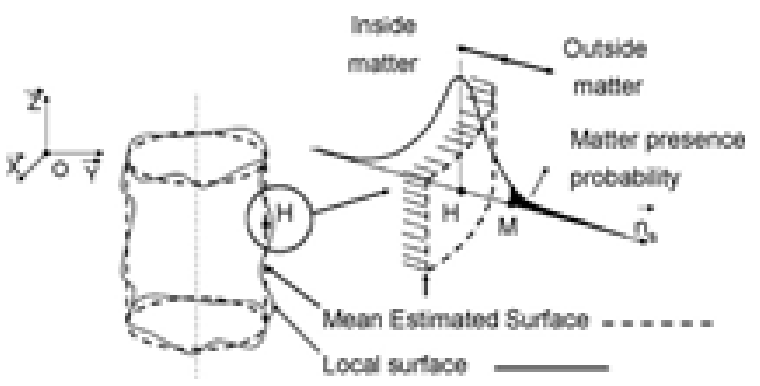

Fig. 5. Probability of presence of the matter. 


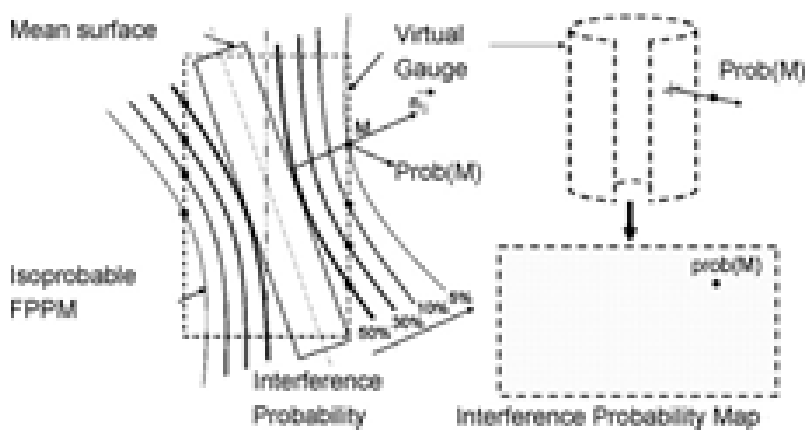

Fig. 6. Matter presence probability.

optimization method is employed for that purpose. The covariance matrix is then derived from the best fit residues calculated at the last optimization step, using a classical variance propagation method.

Our goal is then to calculate the probability of any point $\mathrm{M}$ to be located inside the matter, i.e. to have a negative algebraic distance (Fig. 5). To perform this calculation, all the uncertainties integrated in the random vector are propagated to the estimated integral surface. For that purpose the projection $\mathrm{H}$ of point $\mathrm{M}$, to the mean integral surface is first computed. The expression of the algebraic distance $d_{M}$ between $M$ and the best-fitted feature is thus easily derived (7):

$\mathrm{d}_{\mathrm{M}} 1 / 4 \mathrm{HM} \mathrm{n}_{\mathrm{S}}^{\mathbf{0}}$

where $\stackrel{!}{\mathrm{n}}_{\mathrm{S}}$ is the normal vector of the integral surface at point $\mathrm{H}$. The classical propagation method is then used to calculate its variance (8):

$\operatorname{var}_{\mathrm{M}} \mathrm{d}_{\mathrm{M}} \mathrm{p} 1 / 4 \mathrm{~J} \operatorname{Cov} \mathrm{P}^{\prime} \mathrm{p} \mathrm{J} T$

where $J$ represents the jacobian vector of scalar $d_{M}$. However, this variance only characterizes the variability of the estimated integral surface. To define the boundaries of the probed feature, the variance of the acquired points, as defined by the mean least squares residues, has also to be added to it (9).

$\mathrm{S}_{\text {Global= }}^{2} \stackrel{n_{\mathrm{S}}}{1 / 4 \mathrm{~J}} \operatorname{Covð\mathrm {P}^{\mathrm {P}}\mathrm {J}^{\mathrm {T}}} \mathrm{PS}_{\text {Residues }}^{2}$

This permits calculating the probability $\operatorname{prob}(\mathrm{M})$ of any point $\mathrm{M}$ to be located inside the matter by using a classical integration of a Gaussian law (Fig. 6). Thus, a three-dimensional scalar function $\operatorname{prob}(\mathrm{M})$ is built called field of probability of presence of the matter.

\section{Interference probability map between the FFPM and the virtual gauge}

\subsection{Interference probability map}

The FPPM is particularly well adapted to virtual gauges. During the checking phase, point $\mathrm{M}$ is just taken as a given point of the virtual gauge. Then the FPPM permits calculating the corresponding probability prob(M) (Fig. 6). This probability represents the risk of the selected point $M$ of the virtual gauge to enter the matter, i.e. the non-conformance risk at this location.

This calculation can be done for any point of the virtual gauge elements. To decrease the calculation time, a meshing of each feature, followed by a linear interpolation, is actually performed. A set of probabilities is thus calculated which allows the construction of a graphic plot called interference probability map.

\subsection{Geometrical verification process}

The geometrical verification based on virtual gauge and FPPM, consists finally in verifying if the probabilities of the whole points

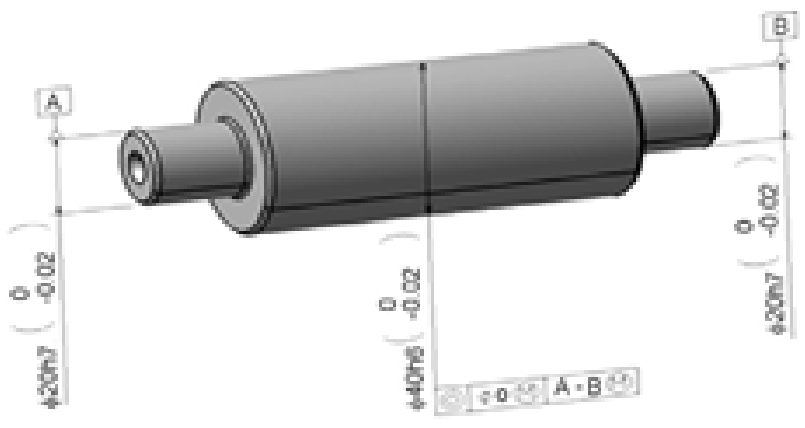

Fig. 7. Gauge definition.

of the calculated IPMs remain below the risk level fixed by the quality control inspector. To maximize the chance of a part to be conforming, the position of the global virtual gauge which minimizes the maximum interference probability must be found. This optimization problem corresponds to a Mini Max problem applied to the whole set of interference probabilities.

\section{Experiment}

Our verification method has been applied to an example. The studied part is a shaft consisting of three cylinders (Fig. 7). The central one has a coaxiality deviation specified in relation to a common datum reference. The specification and the datum reference have a MMC modifier. According to ISO standard, this specification leads to construct three coaxial elementary virtual gauges. The diameters, at the maximum material condition, are respectively $20 \mathrm{~mm}$ for the two datum reference features and $40 \mathrm{~mm}$ for the specified cylinder.

The measurement of the three cylinders has been realized on a classical Coordinate Measuring Machine (CMM). Each cylinder has been probed with 30 points. Two surface models have been fitted to these points. The first one corresponds to a perfect cylindrical integral feature. The second one is a surface of revolution created by a second-order polynomial generatrix which characterizes the major part of the form defects [12]. According to the verification method previously proposed, six different IPMs have been calculated and plotted, i.e. three for the cylindrical model and three for the polynomial one. To simplify the analysis, only the IPM of the central cylinder will be displayed. In order to highlight the limitations of the two different models, three virtual gauge sizes have been considered for the specified feature. The tested diameters were respectively fixed to $40 \mathrm{~mm}, 39.995 \mathrm{~mm}$ and $39.990 \mathrm{~mm}$.

The major fitting results obtained by approximating the acquired surfaces by perfect cylinders are presented in Table 1. In this case, the form defects evaluated on the datum features and the specified surface are large.

Fig. 8 shows the IPM of the central cylinder. On this plot, the interference probabilities are represented by levels of gray where black characterizes the maximum interference risk and white the minimum one. The highest probability of interference between the virtual gauge and the real surface is also given on the left of the figure. Even for the gauge of largest diameter $(40 \mathrm{~mm})$ this value exceeds $10 \%$. The tested part would therefore be rejected. Similar interference probability levels were also calculated for the datum features. The dissymmetry observed on the IPM is due to the optimization step which balances the system of virtual gauges. The

Table 1

Best fit results of the cylindrical model

\begin{tabular}{llll}
\hline & Diameter $(\mathrm{mm})$ & Form defect $(\mathrm{mm})$ & Residue $(\mathrm{mm})$ \\
\hline Datum A & 19.988 & 7.9 & 2.1 \\
Datum B & 19.991 & 8.5 & 2.2 \\
Cylinder & 39.977 & 4.9 & 1.5 \\
\hline
\end{tabular}




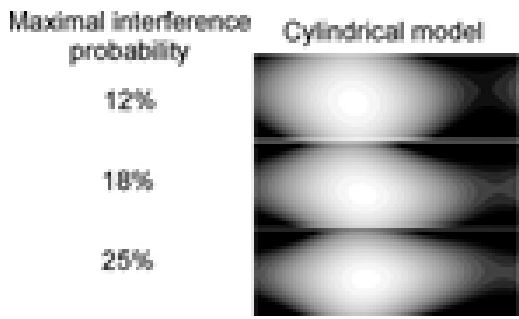

Virtual gauge

diameter

040.000

039.995

039.990

Fig. 8. Specified surface IPM.

Table 2

Best fit results of the polynomial model

\begin{tabular}{llll}
\hline & Diameter $(\mathrm{mm})$ & Deviation $(\mathrm{mm})$ & Residue $(\mathrm{mm})$ \\
\hline Datum A & 19.988 & 1.4 & 0.41 \\
Datum B & 19.991 & 1.7 & 0.47 \\
Cylinder & 39.977 & 1.7 & 0.46 \\
\hline
\end{tabular}

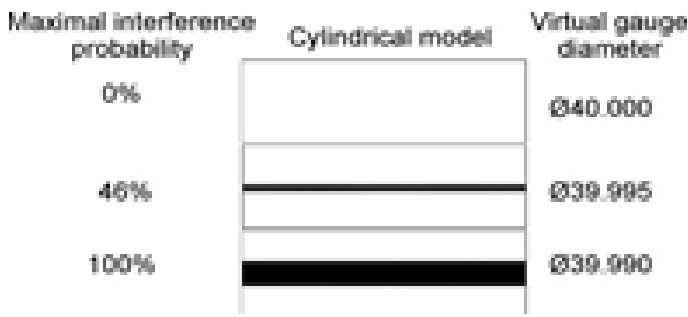

Fig. 9. Specified surface IPM.

global aspect of the IPM does not change when the gauge diameter is shrinking because the uncertainties estimated for the integral surface are large. In fact, the cylindrical model does not account for predominant form defects. Such ignored systematic deviations are therefore considered as measurement uncertainties.

The major fitting results obtained in using the polynomial model are given in Table 2. The standard deviations of the best fit residues are now five times lower than the values derived from the previous approach. These uncertainties decrease because the polynomial model incorporates new information about the form defect [13].

Fig. 9 shows the IPF of the central cylinder. The polynomial model permits accurately localizing where interferences between the virtual gauge and the matter may exist. When the diameter of the virtual gauge decreases the central cylinder becomes more and more constraint. It then forces the global gauge system to rotate and translate during the optimization procedure in order to minimize the maximum interference probability.

For a gauge diameter of $40 \mathrm{~mm}$, the interference probability is close to $0 \%$. Hence, for this specification, the part can be accepted without any doubt. The same kind of results was also found for the datum features IPMs.

\section{Conclusion}

In this paper a new approach has been proposed to verify geometrical specifications. This method, based on virtual gauges includes the measurement uncertainties directly in a global checking process. The probability of any point to be located inside the part has therefore been evaluated, thus defining a field of probability of presence of matter. The FPPM avoids conformance tests based on series of constructions where the uncertainties are estimated after calculation of the geometrical deviations to test. This paper brings to the fore that increasing the number of parameters used to describe and fit the probed surfaces leads to a better characterization of the form defects and thus reduces considerably the uncertainties. In practice, the number of variables of the surface model will however be limited by the quantity of points being acquired.

\section{References}

[1] Weckenmann A, Lorz J, Beetz S (2005) Monitoring Coordinates Measurin Machine by User-defined Calibrated Parts. Proceedings of the Ninth CIRF Seminar on Computer Aided Tolerancing, . (CD-Rom).

[2] Schwenke H, Siebert BRL, Waldele F, Kunzmann H (2000) Assessment o Uncertainties in Dimensional Metrology by Monte Carlo Simulation. Proposal of A Modular and Visual Software. Annals of CIRP 49:395-398.

[3] Balsamo A, Di Ciommo M, Mugno R, Rebaglia BI, Ricci E, Grella R (1999) Evaluation of CMM Uncertainty though Monte Carlo Simulations. Annals of CIRP 48:429-432.

[4] Sommer KD, Siebert BRL, Kochsiek M, Weckenmann A (2005) A Systematic Approach to the Modelling and Uncertainty Analysis of Measurement. Proceedings of the 12th International Metrology Congress, . (CD-Rom).

[5] Srinivasan V (1999) A Geometrical Product Specification Language Based on a Classification of Symmetry Groups. Computer-Aided Design 31:659-668.

[6] Bachmann J, Linares JM, Sprauel JM, Bourdet P (2004) Aide in Decision-making: Contribution to Uncertainties in Three-dimensional Measurement. Precision Engineering 28:78-88.

[7] Pairel E (1997) The Gauge model: A New Approach for Coordinate Measurement. Proceedings of the XIV IMEKO World Congress, 278-283.

[8] Dantan JY, Ballu A (1999) Functional and Product Specification by Gauge with Internal Mobilities. Proceedings of the Sixth CIRP Seminar on Computer-Aided Tolerancing, 37-46.

[9] Clément A, Rivière A, Serré P (1999) Global Consistency of Dimensioning and Tolerancing. Proceedings of the Sixth CIRP International Seminar on ComputerAided Tolerancing, 1-26.

[10] Serré P, Rivière A, Clément A (2005) Dependence and Independence of Variations of Geometric Object. Selected Conference Paper from the Ninth CIRF Computer-Aided Tolerancing Seminar, 23-34.

[11] Sprauel JM, Linares JM, Bourdet P (2001) Contribution of Nonlinear Optimization to the Determination of Measurement Uncertainties. Proceedings of the Seventh CIRP Seminar on Computer-Aided Tolerancing, 235-244.

[12] Summerhays KD, Henke RP, Balwin JM, Cassou JM, Brown CW (2002) Optimizing Discrete Point Sample Patterns and Measurement Data Analysis o Internal Cylindrical Surfaces with Systematic Form Deviations. Precision Engineering 26:105-121.

[13] Estler WT (1999) Measurement as Inference: Fundamental Ideas. Annals of the CIRP 48(2):611-632. 